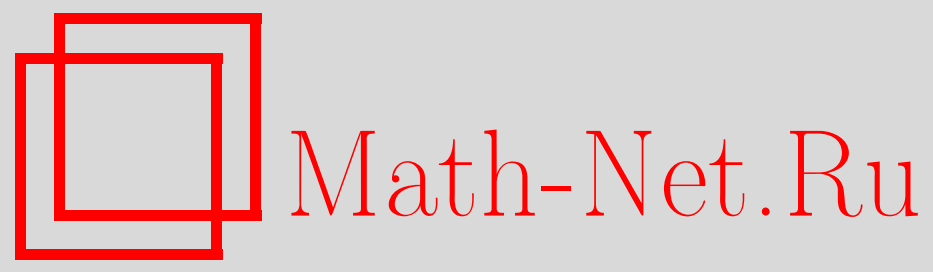

Д. Ю. Черухин, О схемах из функциональных элементов конечной глубины ветвления, Дискрет. матем., 2006, том 18, выпуск 4, 73-83

DOI: https://doi.org/10.4213/dm73

Использование Общероссийского математического портала Math-Net.Ru подразумевает, что вы прочитали и согласны с пользовательским соглашением http: //www . mathnet.ru/rus/agreement

Параметры загрузки:

IP: 52.90 .164 .192

26 апреля 2023 г., 10:32:34

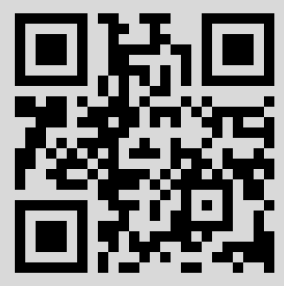


УдК 519.7

\title{
О схемах из функциональных элементов конечной глубины ветвления
}

\author{
() 2006 г. Д. Ю. Черухин
}

В работе введено понятие глубины ветвления схемы из функциональных элементов и рассмотрены классы схем, имеющие глубину ветвления, ограниченную константой. В данных классах схем и различных базисах получены верхние и нижние оценки сложности линейной булевой функции. Построены бесконечно убывающие последовательности мер сложности при фиксированном базисе и растущей глубине ветвления и при фиксированной глубине ветвления и меняющемся базисе.

Работа выполнена при поддержке Российского фонда фундаментальных исследований, проект 05-01-00994, программой «Университеты России», грант УР.04.02.528, и программой Президента Российской Федерации поддержки ведущих научных школ, грант НШ 1807.2003.1.

Базисом будем называть конечную полную систему булевых функций. Определения схемы из функциональных элементов (СФЭ) и формулы в базисе $B$ можно найти, например, в [1]. Скажем, что вершина $v$ схемы $S$ обладает ветвлением, если функция, вычисленная в вершине $v$, используется в дальнейшем вычислении не менее двух раз, а именно, либо вершине $v$ соответствуют не менее двух выходов схемы, либо $v$ является выходом схемы и существует хотя бы одно ребро, выходящее из $v$, либо из $v$ выходит не менее чем два ребра. Вершину $v$ назовем узловой, если либо $v$ является входом схемы, либо $v$ обладает ветвлением.

Глубиной ветвления схемы $S$ называется наибольшее число узловых вершин, содержащихся в одном ориентированном пути в схеме $S$. Таким образом, глубина ветвления формул равна 1 (заметим, что если не считать входы узловыми вершинами, то глубина ветвления бесповторных (см. [2]) формул равна нулю; однако такое определение в некотором смысле неудобно), глубина ветвления схем из функциональных элементов неограничена. В данной работе рассмотрены классы схем, в которых глубина ветвления ограничена константой.

При реализации почти всех булевых функций рассматриваемые классы схем (если глубина ветвления ограничена числом, не меньшим 2) ведут себя подобно схемам из функциональных элементов (а именно, имеют тот же порядок роста функции Шеннона). В настоящей работе показано, что при реализации отдельных функщий, например, линейной функции, данные классы схем более напоминают формулы, а именно, и верхние (теорема 1) и нижние (теорема 2) оценки сложности извлекаются из соответствующих методов оценки сложности формул.

При получении нижних оценок сложности используется традиционный метод забивания переменных, впервые использованный Б. А. Субботовской в [3] для базиса $\{\&, \vee, \neg\}$, 
и распространенный затем на класс обобщенно-монотонных базисов, то есть базисов, в которых линейная функщия имеет нелинейную сложность [4-7]; метод забивания известен также как метод сжатия формул под действием случайной подстановки $[7,8]$. Вопрос о том, применимы ли к схемам ограниченной глубины ветвления аналоги других методов получения нижних оценок сложности формул [9], остается открытым.

Скажем, что мера сложности $L_{1}$ булевых функций предшествует [2] мере сложности $L_{2}\left(L_{1} \leqslant L_{2}\right)$, если $L_{1}(f)=\mathcal{O}\left(L_{2}(f)\right)$ при nesí $\left.f\right) \rightarrow \infty$, где $\operatorname{nes}(f)-$ число существенных переменных функции $f$. Отношение предшествования мер сложности есть предпорядок. Оно порождает отношения строгого предшествования < и эквивалентности мер сложности. Для каждого базиса $B$ и числа $k \geqslant 1$ мы введем меру сложности $L_{B}^{k}$, соответствующую классу схем в базисе $B$, глубина ветвления которых не превосходит $k$. Мы построим (теорема 3) бесконечные последовательности вида $L_{B}^{k_{1}}>L_{B}^{k_{2}}>\ldots$ для каждого обобщенно-монотонного базиса $B$ и вида $L_{B_{1}}^{k}>L_{B_{2}}^{k}>\ldots$ для каждого $k \geqslant 2 ;$ при $k=1$ такие последовательности известны (см., например, [6]). Кроме того, используя улучшенную технику сжатия формул в базисе $B_{0}=\{\&, \vee, \neg\}$ (см. [8]), покажем (теорема 4), что $L_{B_{0}}^{1}>L_{B_{0}}^{2}>\ldots$

Стоит отметить связь глубины ветвления с глубиной в обычном смысле, то есть с наибольшей из длин ориентированных путей в схеме. Схемы глубины ветвления $k$ в базисе $B$ можно рассматривать как схемы глубины $k$ над бесконечным базисом, состоящим из всех функций, бесповторно выразимых [2] в базисе $B$. Вопрос о сравнении мер сложности одной и той же модели схем с разными константными ограничениями на глубину был впервые исследован в [10]: для класса монотонных формул было показано, что при увеличении глубины на единицу мера сложности строго уменьшается.

В настоящее время имеется достаточно много работ по нижним оценкам сложности схем ограниченной глубины; в некоторых из них получены экспоненциальные нижние оценки сложности [9]. В отличие от многих классов схем ограниченной глубины рассматриваемые в данной работе классы являются “сильными”, так как они не слабее класса формул в полном базисе, традищионно считающегося “сильным": наибольшие известные нижние оценки сложности формул не превосходят полинома от числа переменных.

Подстановкой констант называется функция, сопоставляющая некоторым переменным константы из множества $\{0,1\}$. Если $f$ - функция, $A$ - подстановка констант, то через $\left.f\right|_{A}$ обозначим функцию, полученную из $f$ фиксацией переменных, входящих в область определения подстановки $A$, соответствующими константами; через $|A|$ обозначим мощность множества определения подстановки $A$. Функция вида $\left.f\right|_{A}$ называется подфункщией функции $f$.

Базис назовем нормальным (см. [11]), если вместе с каждой функцией он содержит все ее подфункиия. Каждый базис $B$ эквивалентен некоторому нормальному базису, а именно, минимальному нормальному базису, включающему $B$. В дальнейшем будем считать, что все рассматриваемые базисы нормальны. Заметим, что нормальный базис содержит отрицание.

Сложностью схемы $S$ будем считать число ребер, выходящих из узловых вершин; сложность будем обозначать через $L(S)$. Нетрудно показать, что данная мера сложности отличается не более чем в константу раз от традиционной меры сложности СФЭ - числа элементов; в то же время рассматриваемая мера сложности является традиционной для формул (число ребер, исходящих из входов формулы, равно числу вхождений в формулу символов переменных). Обозначим через $L_{B}^{k}(f)$ сложность булевой функщии $f$ в классе СФЭ в базисе $B$, глубина ветвления которой не превосходит $k$. В частности, $L_{B}^{1}(f)-$ сложность функции $f$ в классе формул в базисе $B$. 
Всюду в работе асимптотические соотношения будем понимать в смысле теории предела по базе. Через будем обозначать асимптотическое равенство функщй, через $\asymp-$ равенство по порядку. Если база не указана явно, подразумевается стремление основного независимого параметра (обычно $n$ ) к бесконечности.

При разбиении множества на части будем употреблять выражения вида: “разбить $X$ на $Y$ примерно равных частей” и “разбить $X$ на части примерно по $Y$ элементов". В первом случае имеется в виду, что размеры частей отличаются не более, чем на единицу. Во втором случае подразумевается, что все части, кроме последней, содержат $Y$ элементов, а последняя - не больше $Y$ элементов.

Введем обозначение

$$
\Lambda_{n}=x_{1} \oplus \ldots \oplus x_{n}
$$

Для любого целого $k \geqslant 1$ введем функцию

$$
\varphi_{k}(x)=\frac{1}{1-(1-1 / x)^{k}}
$$

Заметим, что если $x>1$, то $\varphi_{1}(x)=x$ и последовательность $\varphi_{1}(x), \varphi_{2}(x), \ldots$, монотонно убывая, стремится к числу 1 .

Теорема 1. Пусть $B-$ базис, $\gamma>1$. Тогда, если при $n \rightarrow \infty$

$$
L_{B}^{1}\left(\Lambda_{n}\right)=\mathcal{O}\left(n^{\gamma}\right)
$$

то для любого $k$ при $n \rightarrow \infty$

$$
L_{B}^{k}\left(\Lambda_{n}\right)=\mathcal{O}\left(n^{\varphi_{k}(\gamma)}\right) .
$$

Доказательство. На первом шаге объединим переменные в группы (которые будем называть 1-группами) примерно по $n_{1}$ переменных в каждой (числа $n_{1}, n_{2}, \ldots, n_{k-1}$ выберем позже). Сумму переменных в каждой 1-группе реализуем формулой сложности $\sigma\left(n_{1}^{\gamma}\right)$. Общая сложность построенных формул есть $O\left(n_{1}^{\gamma} n / n_{1}\right)$. На втором шаге объединим 1 групшы в более крупные 2-группы, а именно, каждую 2-группу образуем примерно из $n_{2}$ 1-групп. Реализуем сумму переменных в каждой 2-группе формулой, используя в качестве входов этой формулы ранее вычисленные суммы для 1-групп. Общая сложность построенных формул на втором шаге есть $O\left(n_{2}^{\gamma} n /\left(n_{1} n_{2}\right)\right)$.

Подобным образом действуем и дальше. На $k$-м шаге объединим все $n$ переменных в одну $k$-группу и вычислим их сумму, опираясь на суммы переменных в $(k-1)$-группах. Пусть $n_{k}-$ число $(k-1)$-групп, тогда

$$
n_{1} n_{2} \ldots n_{k} \sim n
$$

Сложность формулы, построенной на $k$-м шаге, есть $\mathcal{O}\left(n_{k}^{\gamma}\right)=\mathcal{O}\left(n_{k}^{\gamma} n /\left(n_{1} n_{2} \ldots n_{k}\right)\right)$. Полученная нами схема имеет глубину ветвления $k$ и реализует функцию $\Lambda_{n}$, поэтому

$$
L_{B}^{k}\left(\Lambda_{n}\right)=\odot\left(\sum_{i=1}^{k} n_{i}^{\gamma} n /\left(n_{1} \ldots n_{i}\right)\right) .
$$

Выберем числа $n_{1}, \ldots, n_{k-1}$ так, чтобы слагаемые в сумме (2) были асимптотически равны. После несложных выкладок получим условия для такого выбора

$$
n_{i+1} \sim n_{i}^{\alpha}, \quad \alpha=\frac{\gamma}{\gamma-1}, \quad i=1, \ldots, k-1 .
$$


Подставляя такие числа в (1), получим, что

$$
n \sim n_{1} n_{1}^{\alpha} \ldots n_{1}^{\alpha^{k-1}}=n_{1}^{\left(\alpha^{k}-1\right) /(\alpha-1)} .
$$

Выразив отсюда $n_{1}$ и подставив его в (2), получим, что

$$
L_{B}^{k}\left(\Lambda_{n}\right)=\mathcal{O}\left(k n_{1}^{\gamma} n / n_{1}\right)=\mathcal{O}\left(n_{1}^{\gamma-1} n\right)=\mathcal{O}\left(n^{(\alpha-1)(\gamma-1) / \alpha^{k}-1+1}\right) .
$$

Наконец, преобразуем показатель степени в последней формуле в (3):

$$
\begin{aligned}
\frac{\alpha-1}{\alpha^{k}-1}(\gamma-1)+1 & =\frac{(\gamma /(\gamma-1)-1)(\gamma-1)}{\alpha^{k}-1}+1 \\
& =\frac{1}{\alpha^{k}-1}+1=\frac{1}{1-(1 / \alpha)^{k}}=\varphi_{k}(\gamma) .
\end{aligned}
$$

Теорема 1 доказана.

Введем меру сложности функщий $L_{B}^{*}$ : если $f$ тождественно равна константе, переменной или отрицанию переменной, то $L_{B}^{*}(f)=0$, в противном случае положим $L_{B}^{*}(f)=L_{B}^{1}(f)$. Пусть $f-$ функция от $n$ переменных, $0 \leqslant m \leqslant n$. Тогда положим

$$
L_{B}^{*}(f ; m)=\frac{1}{2^{n-m}\left(\begin{array}{c}
n \\
m
\end{array}\right)} \sum_{A,|A|=n-m} L^{*}\left(\left.f\right|_{A}\right)
$$

(здесь и далее сумма берется по подстановкам $A$, область определения которых содержится в множестве переменных функции $f)$. Другими словами, $L_{B}^{*}(f ; m)-$ сложность случайной подфункщии от $m$ переменных функции $f$. Скажем, что базис $B$ обладает экспонентой сжатия $\gamma$, если при $n=\operatorname{nes}(f) \rightarrow \infty, m \geqslant 1, m=o(n)$, справедливо соотношение

$$
L_{B}^{*}(f)=\Omega\left((n / m)^{\gamma}\right) L_{B}^{*}(f ; m)
$$

Теорема 2. Пусть базис В обладает экспонентой сжатия $\gamma, \gamma>1$. Тогда для любого $k$ npu $n \rightarrow \infty$

$$
L_{B}^{k}\left(\Lambda_{n}\right)=\Omega\left(n^{\varphi_{k}(\gamma)}\right)
$$

Доказательство. Проведем индукцию по $k$. В качестве базиса индукции возьмем случай $k=1$. При $k=1$, утверждение следует из (4), если положить $m=2$.

Проведем индуктивный переход от $k-1$ к $k$. Пусть $S$ - схема минимальной сложности для функции $\Lambda_{n}$ в классе схем глубины ветвления, не большей $k$. Пусть $V-$ множество узловых вершин схемы $S$, отличных от входов. Если $V=\varnothing$, то схема $S$ является формулой и доказываемое утверждение следует из базиса индукции и неравенства $\varphi_{k}(\gamma)<\gamma$.

Рассмотрим случай $V \neq \varnothing$. Вершину $v \in V$ назовем минимальной в $V$, если не существует вершины $v^{\prime} \in V, v^{\prime} \neq v$, такой, что из $v^{\prime}$ в $v$ ведет некоторый ориентированный путь. Пусть $V^{\prime}=\left\{v_{1}, \ldots, v_{r}\right\}-$ множество всех минимальных в $V$ вершин. Пусть также $f_{i}$ - функция, вычисляемая в вершине $v_{i}, 1 \leqslant i \leqslant r$ ( $f_{i}$ зависит от входных переменных схемы $S$ ). Тогда часть схемы $S$, предшествующая вершине $v_{i}$, то есть часть, в которой 
вычисляется функция $f_{i}$, является формулой; обозначим ее через $F_{i}$. Формулы $F_{1}, \ldots, F_{r}$ не содержат общих вершин, за исключением входов, и минимальны, поэтому

$$
L(S) \geqslant \sum_{i=1}^{r} L\left(F_{i}\right)=\sum_{i=1}^{r} L_{B}^{1}\left(f_{i}\right) .
$$

Если

$$
\sum_{i=1}^{r} L_{B}^{1}\left(f_{i}\right) \geqslant n^{\varphi_{k}(\gamma)},
$$

то, в силу (5), теорема доказана. Если же (6) не выполнено, то с учетом (4) для некоторой константы $C$ при $m \geqslant 1, m=o(n)$ справедливы соотношения

$$
\begin{aligned}
n^{\varphi_{k}(\gamma)} & >\sum_{i=1}^{r} L_{B}^{1}\left(f_{i}\right) \geqslant \sum_{i=1}^{r} L_{B}^{*}\left(f_{i}\right) \geqslant C\left(\frac{n}{m}\right)^{\gamma} \sum_{i=1}^{r} L_{B}^{*}\left(f_{i} ; m\right) \\
& =C\left(\frac{n}{m}\right)^{\gamma} \frac{1}{2^{n-m}\left(\begin{array}{l}
n \\
m
\end{array}\right)} \sum_{A,|A|=n-m} \sum_{i=1}^{r} L_{B}^{*}\left(\left.f_{i}\right|_{A}\right) .
\end{aligned}
$$

Существует такая подстановка констант $A,|A|=n-m$, что

$$
n^{\varphi_{k}(\gamma)}>C\left(\frac{n}{m}\right)^{\gamma} \sum_{i=1}^{r} L_{B}^{*}\left(\left.f_{i}\right|_{A}\right)
$$

Положим $m=D n^{\alpha}$, где $\alpha$ удовлетворяет условию

$$
\varphi_{k}(\gamma)-\gamma(1-\alpha)=\alpha,
$$

величину $D$ определим ниже. Перепишем (7) в виде

$$
\sum_{i=1}^{r} L_{B}^{*}\left(\left.f_{i}\right|_{A}\right)<\frac{D^{\gamma}}{C} n^{\varphi_{k}(\gamma)-\gamma(1-\alpha)}=\frac{D^{\gamma}}{C} n^{\alpha}=\frac{D^{\gamma-1}}{C} m .
$$

Константу $D$ выберем так, чтобы выполнялось неравенство

$$
\sum_{i=1}^{r} L_{B}^{*}\left(\left.f_{i}\right|_{A}\right)<\frac{m}{2}
$$

Пусть $M$ - множество переменных, каждая из которых существенна хотя бы для одной из функщй $\left.f_{i}\right|_{A}$, существенно зависящих не менее, чем от двух переменных. В силу (9) справедливо неравенство $|M|<m / 2$. Пусть $A^{\prime}$ - подстановка констант, определенная на множестве $M$. Тогда каждая из функщий $\left.f_{i}\right|_{A \cup A^{\prime}}$ тождественно равна либо константе, либо переменной, либо отрицанию переменной. Осуществим подстановку констант $A \cup A^{\prime}$ в схему $S$ и проведем естественные упрощения, в частности, если функция $\left.f_{i}\right|_{A \cup A^{\prime}}$ равна переменной, то отождествим вершину $v_{i}$ с соответствующим входом, если $\left.f_{i}\right|_{A \cup A^{\prime}}$ равна отрицанию переменной, то поступим аналогично, но предварительно пронесем отрицание через вершину $v_{i}$ (возможно, увеличив число элементов отрицания, что не влияет на сложность); наконец, если функщия $\left.f_{i}\right|_{A \cup A^{\prime}}$ тождественно равна константе, то вершину 
$v_{i}$ удалим, осуществив дальнейшие подстановки этой константы. В результате получим схему $S^{\prime}$, реализующую линейную функцию (или ее отрицание) от не менее чем $m / 2$ переменных. В схеме $S^{\prime}$ каждая из вершин, входящих в множество $V^{\prime}$, либо отсутствует, либо совпадает с одним из входов. Заметим, что любой ориентированный путь П в исходной схеме $S$, содержащий ровно $k$ узловых вершин, содержит хотя бы одну из вершин множества $V^{\prime}$. Действительно, пусть $v$ - первая узловая вершина пути П, отличная от входов. Тогда существует вершина $v^{\prime} \in V^{\prime}$, предшествующая вершине $v$. Заменив в П начальный отрезок так, чтобы путь проходил через вершину $v^{\prime}$, получим путь, содержащий больше $k$ узловых вершин. Получаем противоречие.

Кроме того, каждая узловая вершина схемы $S^{\prime}$ является узловой для схемы $S$. Поэтому глубина ветвления схемы $S^{\prime}$ не превосходит $k-1$.

Применив предположение индукщии, получим (заметив также, что сложности функции и ее отрицания равны) оценку

$$
\begin{aligned}
L(S) \geqslant L\left(S^{\prime}\right) & \geqslant L_{B}^{k-1}\left(\Lambda_{m / 2}\right)=\Omega\left((m / 2)^{\varphi_{k-1}(\gamma)}\right) \\
& =\Omega\left(n^{\alpha \varphi_{k-1}(\gamma)}\right) .
\end{aligned}
$$

Выразив $\alpha$ из (8) и преобразовав показатель степени в (10), получим равенство

$$
\alpha \varphi_{k-1}(\gamma)=\frac{\gamma-\varphi_{k}(\gamma)}{\gamma-1} \varphi_{k-1}(\gamma)
$$

Покажем, что последнее выражение в (11) равно $\varphi_{k}(\gamma)$, что равносильно равенству

$$
\varphi_{k}(\gamma)=\frac{\gamma \varphi_{k-1}(\gamma)}{\varphi_{k-1}(\gamma)+\gamma-1}
$$

Воспользовавшись равенством

$$
\varphi_{k}(\gamma)=\frac{\gamma^{k}}{\gamma^{k}-(\gamma-1)^{k}}
$$

получим, что

$$
\begin{aligned}
\frac{\gamma \varphi_{k-1}(\gamma)}{\varphi_{k-1}(\gamma)+\gamma-1} & =\frac{\gamma \gamma^{k-1} /\left(\gamma^{k-1}-(\gamma-1)^{k-1}\right)}{\gamma^{k-1} /\left(\gamma^{k-1}-(\gamma-1)^{k-1}\right)+\gamma-1} \\
& =\frac{\gamma^{k}}{\gamma^{k-1}+(\gamma-1)\left(\gamma^{k-1}-(\gamma-1)^{k-1}\right)} \\
& =\frac{\gamma^{k}}{\gamma^{k}-(\gamma-1)^{k}}=\varphi_{k}(\gamma) .
\end{aligned}
$$

Равенство (12), а вместе с ним и теорема 2, доказаны.

Функция $f$ называется обобщенно-монотонной, если $f$ по каждому своему аргументу либо не возрастает, либо не убывает. Базис, состоящий только из обобщенно-монотонных функций, называется обобщенно-монотонным. Порядком базиса $B$ называется наибольшее число аргументов у функщий из $B$.

Лемма 1. Обобщенно-монотонный базис В порядка $k$ обладает экспонентой сжатия

$$
\gamma=\frac{3 k-3}{3 k-4}
$$


Доказательство. Будем использовать терминологию и результаты работы [11]. В [11] введена мера сложности формул $P$ такая, что

$$
P(F)=L(F)+M(F)
$$

где $M(F)$ - сумма весов функций, входящих в формулу; вес функции от $l$ аргументов равен $(l-2) / 2$ при $l \geqslant 2$ и 0 при $l \leqslant 1$. Для меры сложности $F$ справедливо неравенство (см. лемму 1 в [11])

$$
L(F) \geqslant \frac{2 k-2}{3 k-4} P(F)
$$

Через $F \uparrow_{A}$ будем обозначать минимальную (с точки зрения меры $P$ ) приведенную формулу, являющуюся упрощением формулы $\left.F\right|_{A}$, полученной, в свою очередь, действием подстановки констант $A$ на формулу $F$.

В лемме 2 в [11] фактически содержится неравенство

$$
P(F)-\frac{P\left(F \uparrow_{y=0}\right)+P\left(F \uparrow_{y=1}\right)}{2} \geqslant \frac{3}{2} L_{y}(F)
$$

где $L_{y}(F)$ - число вхождений в $F$ символов переменной $y$, справедливое для любых приведенной формулы $F$ и переменной $y$. Просуммировав (14) по всем $n$ переменным формулы $F$, разделив на $n$ и применив (13), получим, что

$$
P(F)-\frac{1}{2 n} \sum_{A,|A|=1} P\left(F \uparrow_{A}\right) \geqslant \frac{3}{2 n} L(F) \geqslant \frac{3 k-3}{(3 k-4) n} P(F)=\frac{\gamma}{n} P(F) .
$$

Отсюда следует, что

$$
P(F) \geqslant \frac{1}{1-\gamma / n} \frac{1}{2 n} \sum_{A,|A|=1} P\left(F \uparrow_{A}\right)
$$

Введем меру сложности функций $P_{B}^{*}$, положив $P_{B}^{*}(f)=P_{B}(f)$, если $\operatorname{nes}(f) \geqslant 2$ и $P_{B}^{*}(f)=0$ в противном случае. Тогда для любой функция $f$ от $n$ аргументов справедливо неравенство

$$
P_{B}^{*}(f) \geqslant \frac{1}{1-\gamma / n} \frac{1}{2 n} \sum_{A,|A|=1} P_{B}^{*}\left(\left.f\right|_{A}\right)
$$

Действительно, если $\operatorname{nes}(f) \leqslant 1$, то (16) следует из определения меры $P_{B}^{*}$, а если $\operatorname{nes}(f) \geqslant 2$, то (16) следует из (15), если в качестве $F$ взять приведенный вид минимальной формулы (в смысле меры $P$ ) для функции $f$. Применив неравенство (16) итеративно $n-m$ раз и считая, что функция $\left.f\right|_{A}$ не зависит от тех переменных, вместо которых были подставлены константы, получим неравенство

$$
P_{B}^{*}(f) \geqslant\left(\prod_{i=m+1}^{n} \frac{1}{1-\gamma / i}\right) \frac{1}{2^{n-m}\left(\begin{array}{l}
n \\
m
\end{array}\right)} \sum_{A,|A|=n-m} P_{B}^{*}\left(\left.f\right|_{A}\right)
$$


Оценим произведение в (17):

$$
\begin{aligned}
\prod_{i=m+1}^{n} \frac{1}{1-\gamma / i} & =\exp \left\{-\sum_{i=m+1}^{n} \ln (1-\gamma / i)\right\} \\
& \geqslant \exp \left\{\sum_{i=m+1}^{n} \frac{\gamma}{i}\right\} \asymp \exp \{\gamma(\ln n-\ln m)\}=\left(\frac{n}{m}\right)^{\gamma} .
\end{aligned}
$$

Поставив данную оценку в (17), с учетом соотношения $P_{B}^{*}(f) \asymp L_{B}^{*}(f)$ получим требуемое неравенство (4). Лемма 1 доказана.

Теорема 3. Для любого обобщенно-монотонного базиса В существует последовательность чисел $k_{1}, k_{2}, \ldots$ такая, что $L_{B}^{k_{1}}>L_{B}^{k_{2}}>\ldots$

Для любого $k=1,2, \ldots$ существует последовательность базисов $B_{1}, B_{2}, \ldots$ такая, чmo $L_{B_{1}}^{k}>L_{B_{2}}^{k}>\ldots$

Доказательство. Докажем первое утверждение теоремы. Положим $k_{1}=1$. Пусть $k_{i}$ уже определено; определим $k_{i+1}$. Согласно лемме 1 , базис $B$ обладает экспонентой сжатия $\gamma>1$. Поэтому, в силу теоремы 2 ,

$$
L_{B}^{k_{i}}\left(\Lambda_{n}\right)=\Omega\left(n^{\varphi_{k_{i}}(\gamma)}\right) .
$$

Последовательность $\varphi_{1}(2), \varphi_{2}(2), \ldots$ стремится к единице, следовательно, найдется такое $l$, что $\varphi_{l}(2)<\varphi_{k_{i}}(\gamma)$. Положим $k_{i+1}=l$. Известно [9], что $L_{B_{0}}^{1}\left(\Lambda_{n}\right)=\mathcal{O}\left(n^{2}\right)$. Отсюда и из неравенства $L_{B}^{1} \leqslant L_{\left.B_{0}\right)}^{1}$, справедливого для любого базиса $B$ (см. [2]), следует, что

$$
L_{B}^{1}\left(\Lambda_{n}\right)=\mathcal{O}\left(n^{2}\right)
$$

Тогда, в силу теоремы 1 и (18),

$$
L_{B}^{k_{i+1}}\left(\Lambda_{n}\right)=O\left(n^{\varphi_{k_{i+1}}(2)}\right)=o\left(n^{\varphi_{k_{i}}(\gamma)}\right)=o\left(L_{B}^{k_{i}}\left(\Lambda_{n}\right)\right)
$$

а значит, $L_{B}^{k_{i+1}} \not L_{B}^{k_{i}}$. Наконец, неравенство $L_{B}^{k_{i+1}} \leqslant L_{B}^{k_{i}}$ справедливо в силу того, что класс схем с бо́льшим ограничением на глубину вложен в класс схем с меньшим ограничением.

Докажем второе утверждение. Так же, как в [6], введем функцию

$$
f_{s}\left(x_{1}, \ldots, x_{s}, y_{1}, \ldots, y_{s}\right)= \begin{cases}1, & \text { если } \sum_{i=1}^{s}\left(x_{i}+y_{i}\right)>s \\ x_{1} \oplus \ldots \oplus x_{s}, & \text { если } \sum_{i=1}^{s}\left(x_{i}+y_{i}\right)=s \\ 0, & \text { если } \sum_{i=1}^{s}\left(x_{i}+y_{i}\right)<s\end{cases}
$$

и образуем базис

$$
B_{s}^{\prime}=B_{0} \cup\left\{f_{s}\right\}
$$

который, очевидно, является обобщенно-монотонным.

В силу тождества

$$
f_{s}\left(x_{1}, \ldots, x_{s}, \bar{x}_{1}, \ldots, \bar{x}_{s}\right) \equiv \Lambda_{s}
$$


несложно предложить метод построения формул для функции $\Lambda_{n}$, дающий при $s \geqslant 2$ оценку

$$
L_{B_{s}^{\prime}}^{1}\left(\Lambda_{n}\right)=\mathcal{O}\left(n^{\log _{s}(2 s)}\right)
$$

А именно, множество переменных нужно разбить на $s$ примерно равных групп, каждую группу на $s$ подгрупп и т. д. Если реализованы суммы переменных в каждой из подгрупп, то сумму переменных в группе можно реализовать, подставив построенные формулы (каждую - по два раза) вместо аргументов функции $f_{s}$. Таким образом, при увеличении числа переменных в $s$ раз сложность формулы увеличивается в $2 s+O(1)$ раз. Отсюда следует (19).

Построим последовательность чисел $s_{1}, s_{2}, \ldots$ и в качестве $B_{i}$ возьмем базис $B_{s_{i}}^{\prime}$, $i=1,2, \ldots$ Положим $s_{1}=2$. Далее, пусть число $s_{i}$ определено; выберем $s_{i+1}$. В силу леммы 1 и теоремы 2 для некоторого числа $\gamma_{i}>1$ выполнено равенство

$$
L_{B_{i}}^{k}\left(\Lambda_{n}\right)=\Omega\left(n^{\varphi_{k}\left(\gamma_{i}\right)}\right)
$$

В качестве $s_{i+1}$ возьмем такое число $s$, для которого $\varphi_{k}\left(\log _{s}(2 s)\right)<\varphi_{k}\left(\gamma_{i}\right)$. Тогда в силу (19) и (20) и теоремы 1

$$
L_{B_{i+1}}^{k}\left(\Lambda_{n}\right)=\mathcal{O}\left(n^{\varphi_{k}\left(\log _{s_{i+1}}\left(2 s_{i+1}\right)\right)}\right)=o\left(n^{\varphi_{k}\left(\gamma_{i}\right)}\right)=o\left(L_{B_{i}}^{k}\left(\Lambda_{n}\right)\right)
$$

а значит, $L_{B_{i+1}}^{k} \not L_{B_{i}}^{k}$. Неравенство $L_{B_{i+1}}^{k} \leqslant L_{B_{i}}^{k}$ следует из того, что при $s<s^{\prime}$ функщия $f_{s}$ может быть получена из $f_{s^{\prime}}$ подстановкой констант вместо некоторых переменных.

Теорема 3 доказана.

Лемма 2. Для любого $\gamma, 1<\gamma<2$, базис В $_{0}$ обладает экспонентой сжатия $\gamma$.

Доказательство. Воспользуемся обозначениями и результатами работы [8]. Пусть $f-$ функция от $n$ переменных,

$$
m=o(n), \quad p=\frac{2 m}{n}, \quad q=\frac{2 p}{1-p} .
$$

Рассмотрим случайную подстановку констант $R_{p}$ : переменные независимо друг от друга получают значения 0,1 с одинаковой вероятностью $(1-p) / 2$ и не получают значение с вероятностью $p$. Пусть $L^{2}(f)$ - математическое ожидание случайной величины $L\left(\left.f\right|_{R_{p}}\right)$ при условии $L\left(\left.f\right|_{R_{p}}\right) \geqslant 2$; введем обозначение $L=L_{B_{0}}^{1}(f)$. Тогда согласно лемме 7.2 из [8]

$$
L^{2}(f) \leqslant \begin{cases}30 q^{2} L(\log L)^{3 / 2}, & \text { если } q \leqslant 1 /(2 \sqrt{L \log L}), \\ 200 q^{2} L(\log (1 / q))^{3 / 2}, & \text { если } 1 / 2 \geqslant q \geqslant 1 /(4 \sqrt{L \log L})\end{cases}
$$

(здесь и далее $\log$ означает логарифм по основанию 2).

Из условия первого случая неравенства (21) следует, что

$$
\log L=O(\log (1 / q)),
$$

поэтому, объединяя оба случая (21) в один и используя цепочку соотношений

$$
q \asymp p \asymp m / n=o(1),
$$


получим, что

$$
L^{2}(f)=O\left(q^{2} \log ^{3 / 2}(1 / q)\right) L=O\left(q^{\gamma}\right) L .
$$

Если дополнительно $\operatorname{nes}(f) \geqslant 2$, то

$$
L_{B_{0}}^{*}(f)=L=\Omega\left((1 / q)^{\gamma}\right) L^{2}(f)=\Omega\left((n / m)^{\gamma}\right) L^{2}(f) .
$$

Введем обозначения

$$
\varphi_{i}=p^{i}(1-p)^{n-i}\left(\begin{array}{l}
n \\
i
\end{array}\right), \quad \Psi_{s}=\sum_{i=0}^{s} \varphi_{i}
$$

Запишем вероятностное определение величины $L^{2}(f)$ в виде конечной суммы и оценим ee:

$$
\begin{aligned}
L^{2}(f) & =\sum_{i=0}^{n} p^{i}\left(\frac{1-p}{2}\right)^{n-i} \sum_{A,|A|=n-i} L_{B_{0}}^{*}\left(\left.f\right|_{A}\right) \\
& =\sum_{i=0}^{n}\left(\begin{array}{l}
n \\
i
\end{array}\right) p^{i}(1-p)^{n-i} L_{B_{0}}^{*}(f ; i) \\
& =\sum_{i=0}^{n} \varphi_{i} L_{B_{0}}^{*}(f ; i) \geqslant \sum_{i=m}^{n} \varphi_{i} L_{B_{0}}^{*}(f ; m) \\
& =\left(1-\Psi_{m-1}\right) L_{B_{0}}^{*}(f ; m) .
\end{aligned}
$$

Заметим, что при $i \leqslant p(n+1)$ (а значит, при $i \leqslant m)$, справедливо неравенство

$$
\frac{\varphi_{i}}{\varphi_{i-1}}=\frac{p}{1-p} \frac{n-i+1}{i} \geqslant 1 \text {. }
$$

Тогда, используя формулу Стирлинга $n ! \asymp(n / e)^{n+1 / 2}$, получим, что при $n \rightarrow \infty, m=$ $o(n), m \rightarrow \infty$

$$
\begin{aligned}
\Psi_{m-1} & \leqslant m \varphi_{m} \asymp m\left(\frac{2 m}{n}\right)^{m}\left(\frac{n-2 m}{n}\right)^{n-m} \frac{(n / e)^{n+1 / 2}}{(m / e)^{m+1 / 2}((n-m) / e)^{n-m+1 / 2}} \\
& \asymp 2^{m}\left(\frac{n-2 m}{n-m}\right)^{n-m} \sqrt{m}=2^{m}\left(1-\frac{m}{n-m}\right)^{n-m} \sqrt{m} \\
& \sim\left(\frac{2}{e}\right)^{m} \sqrt{m} .
\end{aligned}
$$

Таким образом, $\Psi_{m-1}$ ограничено бесконечно малой величиной, зависящей только от $m$, а значит, существует такое $m_{0}$, что при $m \geqslant m_{0}$ выполнено неравенство $\Psi_{m-1} \leqslant 1 / 2$. Тогда из (22) и (23) следует, что требуемое неравенство (4) выполнено при $m \geqslant m_{0}$. Наконец, уменьшив константу в правой части (4) и воспользовавшись неравенством $L_{B_{0}}^{*}\left(f ; m_{0}\right) \geqslant L_{B_{0}}^{*}(f ; m)$ при $m \leqslant m_{0}$, добьемся того, чтобы (4) было выполнено при $1 \leqslant m \leqslant m_{0}$. Лемма 2 доказана.

Теорема 4. Справедливы неравенства $L_{B_{0}}^{1}>L_{B_{0}}^{2}>\ldots$ 
Доказательство. Из теорем 1,2 , леммы 2 , оценки $L_{B_{0}}^{1}\left(\Lambda_{n}\right)=\mathcal{O}\left(n^{2}\right)$ (см. [9]) и непрерывности по $x$ функции $\varphi_{k}(x)$ в точке $x=2$ следует, что для любого $k$ и любой константы $c>0$ справедливы соотношения

$$
L_{B_{0}}^{k}\left(\Lambda_{n}\right)=\Omega\left(n^{\varphi_{k}(2)-c}\right), \quad L_{B_{0}}^{k}\left(\Lambda_{n}\right)=\mathcal{O}\left(n^{\varphi_{k}(2)}\right) .
$$

Поэтому в силу строгого убывания последовательности $\varphi_{1}(2), \varphi_{2}(2), \ldots$ для любого фиксированного $k=1,2, \ldots$

$$
L_{B_{0}}^{k+1}\left(\Lambda_{n}\right)=o\left(L_{B_{0}}^{k}\left(\Lambda_{n}\right)\right) \text {. }
$$

Неравенства

$$
L_{B_{0}}^{k+1} \leqslant L_{B_{i}}^{k}, \quad k=1,2, \ldots
$$

очевидны. Теорема 4 доказана.

Автор выражает благодарность рецензенту, сделавшему ряд полезных замечаний, в частности, указавшему на статью [12], в которой были введены аналогичные классы схем из функциональных элементов.

\section{Список литературы}

1. Лупанов О. Б., Асимптотические оченки сложности управляючих систем. Изд-во МГУ, Москва, 1984.

2. Субботовская Б. А., О сравнении базисов при реализации функций алгебры логики формулами. ДАН СССР (1963) 149, №4, 784-787.

3. Субботовская Б. А., О реализации линейных функций формулами в базисе $\vee, \&,-$. ДАН СCCP (1961) 136, №3, 784-787.

4. Мучник Б. А., Оценка сложности реализации линейной функции в некоторых базисах. Киберпетика (1970), №4, 29-38.

5. Перязев Н. А., Сложность представлений булевых функций формулами в немонолинейных базисах. Дискретная математика и ииформатика. Изд-во Иркут. ун-та, Иркутск, 1995, вып. 2.

6. Черухин Д. Ю., О сложности реализации линейной функции формулами в конечных булевых базисах. Дискретиая математика (2000) 12, №1, 135-144.

7. Chockler H., Zwick U., Which bases admit non-trivial shrinkage of formulae? Computational Complexity (2001) 10, №1, 28-40.

8. Håstad J., The shrinkage exponent of de Morgan formulas is 2. SIAM J. Comput. (1998) 27, 48-64.

9. Нигматуллин Р. Г., Сложность булевых фуикиий. Наука, Москва, 1991.

10. Лупанов О. Б., О влиянии глубины формул на их сложность. Кибернетика (1970), №2, 46-49.

11. Черухин Д. Ю., Сверхквадратичные нижние оценки сложности формул в некоторых базисах. Дискретный анализ и исследование операчий. Сер. 1 (2000) 7, №2, 86-95.

12. Ложкин С. А., Оценки высокой степени точности для сложности управляющих систем из некоторых классов. Матем. вопросы кибериетики (1996) 6, 189-214.

Статья поступила 15.03.2005. 